Franciska SKUTTA

Université de Debrecen

\title{
MARGINALITÉ LEXICALE ET MARGINALITÉ GRAMMATICALE DANS LES TEXTES LITTÉRAIRES
}

\section{Introduction}

La présente étude propose une réflexion sur la notion de «périphérie », ou de «marginalité » ${ }^{1}$ au niveau de l'usage de la langue française. Usage particulier qu'est la littérature, chère aux membres de l'École de Prague, comme aux Formalistes russes, et qui nous amène d'emblée dans un domaine en quelque sorte «marginal» de la communication linguistique. Cependant, la notion de « marginalité » apparaîtra ici à un double titre : d'une part, les textes soumis à l'analyse s'opposent, par leur «littérarité », à l'usage pratique ou, si l'on veut, «central » de la langue; d'autre part, au sein de la littérature, ces textes, d'une facture non conventionnelle, constituent des phénomènes plus spécifiquement marginaux, et se placent ainsi consciemment loin d'un certain idéal «classique » d'expression «claire » et « transparente ». Le choix du corpus - deux recueils de petits textes en prose - a été motivé par la présence, et même une forte dominance, dans les textes, d'un type de marginalité linguistique aux dépens de l'autre type : ainsi, la marginalité lexicale sera-t-elle représentée par des extraits de Constellations (1958) d'André Breton, ${ }^{2}$ tandis que la marginalité grammaticale sera étudiée à travers quelques passages de Tardifs instantanés (1987) de JeanLoup Trassard. ${ }^{3}$ L'analyse comparée de ces œuvres a pour objectif de montrer que malgré les procédés hors norme qui les caractérisent, ces textes gardent une certaine cohérence qui leur est propre.

\section{Marginalité lexicale}

Par ce type de marginalité, on pourrait certes entendre l'utilisation de mots ou de locutions qui ne font pas partie du vocabulaire courant du langage standard, et même cultivé, et qui, dans un énoncé ayant un vocabulaire «ordinaire », apparaîtraient ainsi comme déviants, ajoutant à la dénotation pure des connotations de tout ordre: précisions relatives aux référents désignés (rareté, formes et fonctionnements spécifiques des objets dénotés), à l' «âge » des mots (archaïsmes et néologismes), à leur provenance géographique (dialectes, emprunts récents gardant leur sonorité et leur graphie étrangères) ou socio-culturelle (langues de spécialités; parlers populaires, argots ou, au contraire, vocabulaire recherché, voire solennel). Bien évidemment, le caractère marginal de telles expressions est largement fonction de leur contexte d'utilisation: des mots comme gnaf ou

\footnotetext{
${ }^{1}$ Ces deux termes seront utilisés ici comme des synonymes, tous les deux impliquant dans leur signification 'un éloignement par rapport au centre'.

${ }^{2}$ In : BRETON André (1999), Signe ascendant, Paris, Gallimard, p. 125-171. L'année 1958 est celle de la rédaction de Constellations, comme c'est indiqué par l'auteur, tandis que la première édition par Pierre Matisse à New York date de 1959.

${ }_{3}^{3}$ Paris, Gallimard. L'analyse portera essentiellement sur la Première partie, intitulée Quoi ? (p. 9$31)$.
} 
chégros seraient d'un usage «central» dans le langage technique de la cordonnerie, mais ils frappent le lecteur non spécialiste qui les rencontre tout au début du premier petit texte de Constellations, intitulé Le lever du soleil, où il n'est autrement pas question du métier de cordonnier :

Il était dit que le jeu de mains devait mal finir. C'en est fait, une bonne fois le canut et le gnaf ont réglé leur compte; on en est quitte pour une tourbe à ne pas démêler la soie du chégros.

(p. 129)

Qui plus est, aucun des deux mots ne figure dans Le Petit Robert, et seul le nom gnaf (substantif masculin) se trouve dans le Trésor de la Langue Française, comme un mot «vieilli et populaire » pour signifier 'cordonnier', 'savetier'. Cependant, dans le texte d'André Breton - texte littéraire d'un français standard et même recherché-, ce n'est pas cet usage «marginal», car stylistiquement « marqué », du mot gnaf qui constitue la principale difficulté pour le lecteur, mais bien plutôt le fait que ce mot semble sémantiquement étranger à son contexte, de sorte que son correspondant standard, le mot cordonnier y paraîtrait tout aussi bizarre. En effet, du moment que le sens de gnaf a été identifié (avec ou sans dictionnaire, selon nos compétences), le caractère marginal de ce mot «vieilli et populaire » sera simplement enregistré, ou peut-être même goûté et apprécié par le lecteur, mais ce n'est pas à ce niveau - celui du choix d'entre les synonymes possibles - que se manifeste le fonctionnement en quelque sorte «marginal », hors norme, de ce mot placé dans un certain contexte. Ainsi serait-il préférable de parler ici d'une "marginalité lexico-sémantique », où certaines incompatibilités sémantiques entre les membres d'une collocation, ou dans la progression thématique du texte peuvent effectivement gêner la compréhension. Par là, les textes comme ceux de Constellations, s'écartent très nettement de la communication utilitaire de tous les jours, et se placent, pour ainsi dire, à la «marge » de nos activités linguistiques habituelles.

On sait que la littérature surréaliste, cultivant l'«écriture automatique », abonde en images quasi indéchiffrables; or, Constellations d'André Breton en constitue un bel exemple. Il s'agit là en fait d'un ensemble de vingt-deux textes courts - d'une prose poétique, comme on s'y attend - inspirés au poète par la série éponyme des gouaches que Joan Miró avait peintes en 1940-1941. Et même s'il est vrai que les proses parallèles de Breton ne constituent pas une simple copie verbalisée des tableaux de Miró, ce cadre pictural ne manque pas d'assurer aux textes une certaine cohérence globale : celle du «miromonde »- « une succession délibérée d'œuvres de même format, empruntant les mêmes moyens matériels d'exécution » (BRETON, 1999 : 127) -, dont les figures tourbillonnantes font naître chez Breton des textes baignés d'une atmosphère de rêve et de poésie. Cependant, au niveau des textes particuliers, la cohérence est plus difficile, sinon parfois

\footnotetext{
${ }^{4}$ Curieusement, le substantif masculin chégros figure dans le Grand dictionnaire français-hongrois (1999) d'ECKHARDT Sándor et OlaH Tibor (Budapest, Akadémiai Kiadó), dans le sens de 'ligneul', 'gros fil enduit de poix à l'usage des cordonniers', mais sans indication d'une valeur stylistique particulière.
} 
impossible, à établir d'une manière rassurante, et cela indépendamment du problème de l'«authenticité » de l'écriture automatique, comme le souligne Michael Riffaterre : «Peu importe [...] que l'écriture automatique soit authentique ou non. Sa littérarité ne consiste pas à être une dictée du subconscient, mais à en avoir l'air» (RIFFATERRE, 1979: 249). Or, cette apparence de spontanéité incontrôlée est bien plutôt le produit d'un «travail de la forme très conscient » (RIFFATERRE, 1979 : 236), car le poète cherche à créer "l'image la plus forte», c'est-à-dire « celle qui présente le degré d'arbitraire le plus élevé [...], celle qu'on met le plus longtemps à traduire en langage pratique » (BRETON, $1985: 50$ ). On peut bien avoir ici l'impression qu'André Breton nous invite exprès à réfléchir sur la relation entre "centre» et "périphérie », tellement l'image arbitraire nous éloigne de l'usage pratique, «central », de la langue, sans pour autant anéantir tout lien entre les deux sphères, lien assuré par une possible "traduction», un essai d'interprétation. Pour en donner un exemple, voici l'un des textes de Constellations cité dans son intégralité :

\section{L'étoile matinale}

Elle dit au berger: «Approche. C'est moi qui t'attirais enfant vers ces caves profondes où la mer en se retirant gare les œufs des tempêtes que lustre le varech, aux myriades de paupières baissées. Seulement à la lumière frisante, comme on met la main sur les superbes fossiles au long de la route qui se cherche dans la montagne dynamitée, tu brûlais de voir jaillir l'arête d'un coffre de très ancien ouvrage qui contînt (ce n'est même pas la peine de le forcer) tout ce qui peut ruisseler d'aveuglant au monde. Je te le donne parce que c'est toi comme chaque jour pour que tes sillons grisollent et que, plus flattée qu'aucune, ta compagne sourie en te retrouvant. » (p. 139)

Dans ce texte rédigé avec une grammaire parfaite, celle du langage littéraire classique (contenant par exemple un subjonctif imparfait en subordonnée relative), certaines collocations insolites, ainsi que la progression thématique étrange constituent tout de même un obstacle à la lecture courante.

Au niveau des collocations, la marginalité lexico-sémantique sera illustrée ici par la collocation eufs des tempêtes, où les signifiés des deux substantifs, apparemment reliés par un rapport de possession, sont décidément incompatibles, du moins pour l'acception première des deux mots. Pour reprendre les paroles de Breton, cette image «présente le degré d'arbitraire le plus élevé » possible ; en revanche, elle peut être traduite en langage pratique, si l'on se laisse guider par ces paroles de Riffaterre :

«Il me semble pourtant que beaucoup de ces images ne paraissent obscures et gratuites que si elles sont vues isolément. En contexte, elles s'expliquent par ce qui précède : elles ont des antécédents plus aisément déchiffrables, auxquels elles sont rattachées par une chaîne ininterrompue d'associations verbales qui relèvent de l'écriture automatique.» (RIFFATERRE, 1979 : 217) 
En effet, dans le contexte plus ou moins immédiat, précédent et subséquent, de la collocation oufs des tempêtes, on trouve certains mots qui sont susceptibles d'aider à l'interprétation de cette association sémantique obscure : les substantifs caves (profondes), mer, varech, (les myriades de) paupières (baissées), et les verbes se retirer, garer, lustrer. Or, il est assez facile d'identifier une première relation, métonymique, entre tempêtes et mer, de sorte que le rapport de possession - ou d'origine - supposé entre $x u f s$ et tempêtes peut être transposé à œufs et mer, plus précisément, à une collocation possible : aufs de la mer. Il s'agirait donc de certains objets «possédés » et contenus par la mer, et qui en émergent lorsque les flots des tempêtes (ou simplement des marées) les projettent à l'extérieur. De plus, par ce mouvement violent, la mer - comme une mère « met au monde» ses œufs, ce sens n'étant pas étranger à un des aspects du signifié du mot $\propto u f$ : 'produit des femelles ovipares'. Ce rapport sémantique entre oufs et mer est renforcé par le sens des verbes se retirer et garer: une fois ce mouvement violent apaisé, la mer se retire tout en garant les $œ u f s$, comme le font les oiseaux ou les poissons, dans des endroits protégés, que sont ici ces caves profondes. Reste à savoir ce que désigne le mot $x u f$ par rapport à sa « mère », la mer, et cette fois, c'est le contexte subséquent qui en donne la solution, notamment grâce à un rapport métaphorique entre les termes oufs et paupières baissées, les deux objets évoqués se ressemblant par leur forme ovale, et peut-être même par leur taille. Certes, ces deux termes pris en eux-mêmes sont encore trop éloignés l'un de l'autre pour que l'on puisse reconnaître le référent de $2 u f s$ (de la mer, donc des tempêtes), et c'est ici que peut nous aider l'expression «[les oeufs des tempêtes] que lustre le varech », évoquant les mouvements ondulants par lesquels cette plante aquatique polit la surface solide de ces objets pour y faire apparaître (un peu à la manière du révélateur de la photographie) des myriades de dessins très fins, de la forme de paupières baissées. Pour peu que l'on connaisse ces objets dans la réalité, on les reconnaît enfin comme étant les coquillages - ressemblant par la forme à la fois aux oufs et aux paupières baissées - que l'enfant allait chercher au lever du jour, mais que le texte - au lieu de les nommer directement par le terme conventionnel des dictionnaires - désigne de cette manière détournée, à travers tout un réseau de relations métonymiques et métaphoriques, obscures, sans doute, et néanmoins identifiables à l'aide du contexte linguistique.

Cette brève analyse d'une seule collocation, dont les deux termes évoquent en fait un seul référent à un point précis du texte, n'épuise pas, loin de là, tout le caractère «marginal » de l'œuvre, car les associations lexico-sémantiques étranges surgissent non seulement à ce niveau, pour ainsi dire, «local », mais aussi au niveau «global » de la progression thématique, une progression apparemment trop rapide pour une interprétation aisée. En effet, cette progression ne peut s'appuyer ici sur une continuité référentielle évidente, assurée normalement par des chaînes anaphoriques qui sont pratiquement inexistantes dans ce texte, ${ }^{5}$ de sorte qu'il

\footnotetext{
${ }^{5}$ A part les quelques anaphores «structurales» (c.-à-d. syntaxiques) assurées par les pronoms relatifs, on ne trouve que trois anaphores «textuelles», elles-mêmes assez vagues d'ailleurs, exprimées par des pronoms personnels : «ce n'est même pas la peine de le forcer » (antécédent : un coffre) ; «je te le donne» (antécédent : un coffre ou tout ce qui peut ruisseler d'aveuglant au monde ?) ; et, avant tout, le pronom elle au début du texte, dont l'antécédent doit être le GN du titre même, l'étoile matinale.
} 
convient de chercher d'autres points d'appui, comme les associations reposant sur certaines isotopies, autrement dit une communauté partielle de sèmes entre termes associés et leur persistance dans le texte. Ainsi peut-on suivre des associations à partir du sens deviné de 'coquillages', en passant par les superbes fossiles jusqu'au coffre de très ancien ouvrage, chacun de ces termes évoquant un 'passé lointain' et le 'passage du temps' - ce dernier étant d'ailleurs rapporté également à la vie personnelle du berger, depuis l'enfance pleine de rêves et d'espoirs de richesses matérielles, de tout ce qui peut ruisseler d'aveuglant au monde, jusqu'à l'âge mûr, peut-être désabusé, mais paisible et serein d'un homme dont les sillons grisollent et que sa compagne retrouve dans la joie. Et pour finir, on reconnaît facilement une autre isotopie, plus persistante même, car traversant l'ensemble des textes de Constellations, celle de la 'lumière' : dans L'étoile matinale - cette lumière amie du berger -, depuis le titre, on trouve des expressions évoquant, directement ou indirectement, l'idée de la lumière, telles que lustrer, la lumière frisante, brûler, aveuglant, mais une «luminosité » semblable envahit plusieurs autres textes aux « titres parlants » très poétiques : Le lever du soleil, Le crépuscule rose caresse les femmes et les oiseaux, Femme à la blonde aisselle coiffant sa chevelure à la lueur des étoiles, Personnages dans la nuit guidés par les traces phosphorescentes des escargots. Quant à cette cohérence globale assurée par l'idée de la lumière, on ne s'en étonne guère: elle doit trouver son origine, du moins en partie, dans l'inspiration picturale, les Constellations de Joan Miró.

\section{Marginalité grammaticale}

Si pour André Breton, « comparer deux objets aussi éloignés que possible l'un de l'autre [...] demeure la tâche la plus haute à laquelle la poésie puisse prétendre »(BRETON, 1955 : 148), tout autre est la «marginalité », l'apparente incohérence de l'écriture dans un texte d'un auteur français contemporain, l'écrivain-photographe Jean-Loup Trassard. Auteur de romans, de nouvelles et d'essais, d'un style classique, Trassard a expérimenté, dans Tardifs instantanés, une écriture, pour ainsi dire, de tension vers les limites du fonctionnement linguistique. Dans ce recueil d'une soixantaine de «micro-récits » et de «microdescriptions » sans titres, évoquant des souvenirs personnels, la première section de dix petits textes, intitulée Quoi ?, constitue un ensemble autonome de «scènes de la vie parisienne », où cette écriture expérimentale est particulièrement frappante ; comme le note Jean-Pierre Richard, Trassard «y invente un discours qui vise à briser toutes les continuités [...] pour cerner la solitude d'un instant, d'un lieu, d'un objet, d'un souvenir » (RICHARD, 1990 : 164). Or, ici, les continuités brisées n'ont pas pour origine des associations lexico-sémantiques lointaines, mais des constructions grammaticales insolites, voire inacceptables selon la grammaire du français courant. De plus, cette «marginalité grammaticale » est loin d'être un phénomène sporadique, avec des agrammaticalités apparaissant par-ci par-là ; tout au contraire, les textes sont envahis de constructions "fautives », brisées et lacunaires. Pour en donner une idée, citons le début du deuxième texte (extrait d'une longueur égale à celle du texte de Breton), où le narrateur, de retour chez lui par un temps pluvieux et 
chargé des provisions pour le dîner, prend un moment de repos avant de monter l'escalier :

Pluie tout le soir, m'avait fait hâter le pas - ma fourrure en devenait trop chaude - rapluie mal fermé qui s'accroche, une des lettres avant la poste tombant plate dans l'eau l'encre l'enveloppe entièrement pleurante, manche tors à tenir mais en plus maintenant chargé, je venais d'y penser dans la rue cherchais entre voitures stationnées à remonter sur le trottoir idiot de me crever le poids des pots de verre cette fois deux kilos de pommes puis dîner pourtant habituel. Dans l'immeuble replie le mouillé pousse vitrage et - ha ! - sur une des premières marches je n'en plus lâche les poignées de mon sac sur tapis rouge tenu par des barres de cuivre pose. (p. 13)

Ce passage, choisi au hasard, offre une présentation condensée de la « grammaire trassardienne », dont la principale caractéristique frappante pourrait être désignée par le terme de discontinuité, terme qui semble assez bien traduire à la fois une impression et un fait réalisé dans le texte : l'impression de désordre empêchant la lecture continue, et les structures effectives produisant cette impression. Ces structures elles-mêmes se laissent ensuite classer selon les types de procédés par lesquels elles sont formées, à savoir la suppression d'éléments de diverses natures et la permutation des éléments présents donnant des séquences où les termes de la phrase sont disloqués par rapport à leurs positions habituelles. Ces phénomènes seront illustrés essentiellement par des exemples relevés dans l'extrait choisi, mais au besoin, d'autres exemples seront pris dans l'ensemble du corpus.

\section{Suppression}

Dans ce domaine, les exemples, fort nombreux, touchent avant tout les unités grammaticales, créant des syntagmes défectueux par l'omission du déterminant :

- Pluie tout le soir ; manche tors à tenir ; entre voitures stationnées ; sur tapis rouge

ou par l'ellipse du pronom personnel sujet conjoint (quelle que soit la personne grammaticale) :

- cherchais [...] à remonter ; replie [...] pousse vitrage ; Savait que nous devions (p. 18).

L'absence fréquente d'éléments de liaison produit des enchaînements de séquences juxtaposées, que ce soit par la suppression de la préposition attendue :

- l'encre [de] l'enveloppe ; achevait [de] ranger des papiers (p. 21); roulé [à la] gare (p. 22)

ou par l'omission des conjonctions, devenant presque « la règle » dans la majeure partie de chacun des textes. 
Tandis que les unités grammaticales supprimées - unités appartenant à des classes fermées - sont relativement faciles à restituer, la suppression, peu habituelle, des unités lexicales, de diverses classes ouvertes, peut provoquer un choc dans la lecture, la restitution de telles unités étant plus incertaine et demandant le recours à un contexte plus large. Certes, parfois les unités omises font partie d'une locution figée que l'on reconnaît aisément (en partie, sans doute, grâce au contexte grammatical) :

- je n'en [peux] plus; elle s'est [mise] debout (p. 21)

mais dans d'autres cas, surtout lorsque l'élément absent est un substantif, seul le contexte, proche ou lointain, rend probable telle ou telle restitution :

- replie le [parapluie] mouillé ; dans l'escalier, j'entrouvre porte, penche sur la...mais (p. 18)

où on peut hésiter entre la rampe et la cage d'escalier, la suite laissant le lecteur dans cette incertitude.

\section{Permutation}

Ce procédé ne consiste pas, on s'en doute bien, en un simple changement d'ordre des mots dans la proposition, mais il produit un bouleversement plus profond. Il arrive notamment que des termes étroitement liés soient placés à une distance presque exagérée :

- Savait que nous devions pour les deux jours ou alors elle de son côté partir (p. 18)

ou que le noyau de la proposition suive, au lieu de les précéder, toute une série de compléments :

- sur une des premières marches je n'en plus lâche les poignées de mon sac sur tapis rouge tenu par des barres de cuivre pose.

Cette dernière solution est particulièrement suggestive quant aux motifs de la permutation, en particulier de cette dislocation extrême du prédicat en position rhématique à la fin d'une phrase déjà assez longue. En effet, ce procédé, en plus d'exprimer une situation, donne une certaine allure à la phrase qui imite le déroulement temporel des événements : après une cascade de petits événements exaspérants, vient enfin un moment de repos et de soulagement, que la structure de la phrase exprime avec insistance, surtout si l'on « entend » derrière le verbe pose, le substantif homophone, pause. Il convient de faire remarquer à ce propos qu'une lecture à haute voix donne à ces textes un intérêt tout particulier auquel la lecture silencieuse ne pourrait nullement atteindre.

Un autre exemple pris dans l'extrait cité :

- cherchais entre voitures stationnées à remonter sur le trottoir 
suggère que, selon les interprétations possibles, un même élément, comme le complément circonstanciel en italique, peut établir un double rapport avec son contexte ; ici par sa dépendance soit de cherchais, soit de remonter sur le trottoir [entre voitures stationnées].

\section{Double valeur d'une unité}

Comme le montre l'exemple ci-dessus, ce phénomène, très fréquent dans le texte de Trassard, semble combiner les deux procédés précédemment analysés, surtout lorsque l'unité en question se trouve à la limite de deux séquences juxtaposées :

- plus loin sans doute le réfectoire des vieux crient heurtant des assiettes (p. 21)

où tout indice de démarcation étant supprimé, l'expression des vieux peut permuter, «aller et venir », entre les deux séquences, soit comme un groupe nominal, sujet de crient, soit comme un groupe prépositionnel, complément de réfectoire avec, dans ce dernier cas, ellipse du sujet devant le verbe crient.

Enfin, ce procédé, fort économique, de doter une unité d'une double valeur va jusqu'à produire des formes linguistiques nouvelles au niveau des unités lexicales, par la troncation d'une unité (provoquée par la rencontre de deux unités de sonorité semblable), procédé accompagné ou non par un télescopage :

- tapis cramoisilencieux (p. 18) (cramoisi - silencieux)

- comptabili-dédale (p. 25) (comptabilité - dédale)

- bouche tendre bien dessi...nez charmant (p. 21) (dessinée - nez).

De ce point de vue, cette troncation (sans télescopage) figurant dans notre extrait :

- Pluie tout le soir, m'avait fait hâter le pas [...] rapluie mal fermé qui s'accroche

constitue un cas exceptionnel, encore plus marginal que le reste, car tandis que pas est d'abord un substantif autonome, plus loin, cette même unité, réduite au statut de syllabe (par son aspect phonétique), sera réutilisée, car intégrée au mot parapluie, lui-même introuvable tel quel dans le texte, mais reconstruit mentalement par une lecture rétroactive, à partir de l'élément tronqué, rapluie.

Cet inventaire, très bref par rapport au nombre élevé des données, doit suggérer l'extraordinaire condensation atteinte par les diverses constructions agrammaticales du texte de Trassard. Or, l'apprentissage graduel de la « grammaire trassardienne » nous fait comprendre qu'une telle condensation ne peut être gratuite : elle est effectivement mise au service de la cohérence des dix petits textes, dont chacun, par cette écriture nerveuse et lacunaire, cherche à reproduire la parole intérieure de quelqu'un qui évoque des souvenirs surgissant pêle-mêle, comme les objets réunis simultanément, au hasard d'une prise de vue imaginaire de ces «tardifs instantanés ». 


\section{Conclusion}

L'analyse comparée de ces deux textes littéraires, d'un degré de «marginalité » assez élevé, met en relief des questions linguistiques et pragmatiques d'ordre général. Tout d'abord, on se rend compte que si chacun des deux textes s'est servi d'un seul type de marginalité, cela doit être le seul chemin viable, car sans la présence d'un noyau «central», d'un appui linguistique solide - la morphosyntaxe parfaite des enchaînements sémantiquement problématiques chez Breton, et en revanche, un vocabulaire simple dans des combinaisons sémantiquement acceptables supportant les agrammaticalités chez Trassard $^{6}-$ le degré de marginalité dépasserait les limites de l'interprétabilité des textes, et même de la patience du lecteur. Se pose ensuite la question de la redondance du message, et des possibilités de restitution en cas de séquences défectueuses, à redondance insuffisante. Il est clair que les restitutions sont plus évidentes dans le domaine des éléments grammaticaux, sans se montrer pour autant absolument univoques dans tous les cas; ainsi peut-on hésiter, lors de l'absence de déterminant, à choisir le défini ou l'indéfini, ce qui amène à réfléchir, à un niveau plus général, sur le problème et l'importance relative d'attribuer au référent le trait sémantique 'défini' $v s$ 'indéfini'. Enfin, quant à la redondance ellemême, l'usage linguistique marginal prouve de manière frappante son rôle indispensable dans la transmission sûre des informations au cours de nos communications ordinaires, bref, la place «centrale » de la redondance parmi les phénomènes linguistiques, et par conséquent, le caractère «périphérique » des messages - tels les deux textes analysés - où la redondance fait défaut.

Cependant, si certains types de textes littéraires arrivent à se construire et à se montrer cohérents malgré leur caractère étrange et dérangeant, ne serait-ce pas, pour ces textes, une mise au «centre » de la «périphérie », et par là - peut-être pour toute littérature - un éloge de la « marginalité »?

\section{BIBLIOGRAPHIE}

Textes analysés

BRETON André (1999), Signe ascendant, Paris, Gallimard, Coll. NRF Poésie.

TRASSARD Jean-Loup (1987), Tardifs instantanés, Paris, Gallimard.

Références

BRETON André (1955), Les vases communicants, Paris, Gallimard.

BRETON André (1985), Manifestes du surréalisme, Paris, Gallimard, Coll. Folio Essais.

RICHARD Jean-Pierre (1990), L'état des choses. Études sur huit écrivains d'aujourd'hui, Paris, Gallimard, Coll. NRF Essais.

RIFFATERRE Michael (1979), La production du texte, Paris, Seuil.

SKUTTA Franciska (2003), Une grammaire de la discontinuité, in : SANCHEZ

MIRET Fernando (ed.), Actas del XXIII Congreso Internacional de Lingüística

${ }^{6}$ Même dans cette grammaire hors norme, il reste des points «intouchables », tels le genre des substantifs, la conjugaison, et l'accord entre sujet et prédicat, substantif et adjectif-épithète. 
y Filología Románica (Salamanca, 2001), Tübingen, Niemeyer, Vol. IV, p. 387-395.

SKUTTA Franciska (2010), La cohérence cachée, in : ILIESCU Maria (éd.), Actes du $X X V^{e}$ Congrès International de Linguistique et de Philologie Romanes (Innsbruck, 2007), Berlin - New-York, De Gruyter, Vol. V, p. 541-548.

\section{SUMMARY}

This paper discusses two contrasting types of linguistic marginality in rather unconventional literary texts. Lexico-semantic incompatibility is analysed in Constellations (1958) by André Breton, whereas a variety of agrammatical constructions are examined in Tardifs instantanés (1987) by Jean-Loup Trassard. The analysis shows that in spite of their non-standard linguistic structures, such texts establish a specific coherence, placing peripheral phenomena in the centre of interest. 